\title{
Nonextensive statistical effects on the nuclear equation of state and on nuclear astrophysical problems
}

\author{
Andrea Lavagno* \\ Politecnico di Torino - Dipartimento di Fisica and INFN Sezione di Torino \\ E-mail: andrea. lavagno@polito.it
}

Following the basic prescriptions of the Tsallis' nonextensive thermodynamics, we study the relativistic nuclear equation of state in the hadronic and in the quark plasma phase. We show that small deviations from the standard extensive statistics, due to long-range and memory interactions between particles inside astrophysical plasma, imply remarkable effects into the shape of the equation of state and, as a consequence, in many high-energy astrophysical problems such as the evolution of a newly-born proton-neutron star after a supernova explosion.

International Symposium on Nuclear Astrophysics - Nuclei in the Cosmos - IX

June 25-30 2006

CERN, Geneva, Switzerland

${ }^{*}$ Speaker. 


\section{Introduction}

It has been shown that the nonextensive generalization of the Boltzmann-Gibbs thermostatistics, proposed by Tsallis, can be very relevant in many physical applications where long-range interactions, long-range microscopic memories and/or fractal space-time constraints are present [1]. In particular, recently, many authors outline the possible connection to the nonextensive statistical framework with nuclear and high energy physical applications [2, 3, 4, 5, 6, 7]. The aim of this work is to generalize the basic concepts of the nonextensive statistical mechanics to the relativistic regime and to investigate, through the obtained relativistic thermodynamic relations, the relevance of nonextensive statistical effects on the hadronic and on the quark-gluon plasma (QGP) equation of state (EOS). As we will see, small deviations from the extensive thermostatistics produce a significant modification into the shape of the hadronic and QGP equation of state with important consequence on the deconfined phase transition and on several nuclear astrophysical problems.

\section{Nonextensive statistics in hadronic matter and QGP}

The motivation of the importance of non-standard statistical effects in nuclear and high energy physics lies in the fact the extreme conditions of density and temperature in high energy nuclear collisions give rise to memory effects and long-range color interactions. These conditions imply the presence of non-Markovian processes in the kinetic equation affecting the thermalization process toward equilibrium as well as the standard equilibrium distribution. A rigorous determination of the conditions that produce a nonextensive behavior, due to memory effects and/or long-range interactions, should be based on microscopic calculations relative to the parton plasma originated during the high energy collisions and non-perturbative QCD effects. Such an ambitious goal lies out the scope of the present paper and, at this stage, we limit ourselves to consider the problem from a qualitative point of view. However, it is noteworthy to notice that in proximity of the hadronicQGP phase transition, non-perturbative QCD calculations become important. Only a small number of partons is present in the Debye sphere: the ordinary mean field approximation of the plasma is no longer correct and memory effects are not negligible.

From the above considerations it appears reasonable that in regime of high density and temperature both hadronic and quark-gluon EOSs must be affected by nonextensive statistical effects. In the next two subsections, we will study the two EOSs separately on the basis on the previously obtained relativistic thermodynamic relations.

\subsection{Nonextensive hadronic equation of state}

Concerning the hadronic phase, we use a relativistic self-consistent theory of nuclear matter in which nucleons interact through the nuclear force mediated by the exchange virtual isoscalar and isovector mesons $(\sigma, \omega, \rho)[11,12]$. On the basis of Ref.[8], the pressure and the energy density can be written as

$$
\begin{gathered}
P=\sum_{i=n, p} \frac{2}{3} \int \frac{\mathrm{d}^{3} k}{(2 \pi)^{3}} \frac{k^{2}}{E_{i}^{\star}(k)}\left[n^{q}\left(k, \mu_{i}^{\star}\right)+n^{q}\left(k,-\mu_{i}^{\star}\right)\right]-\frac{1}{2} m_{\sigma}^{2} \phi^{2} \\
-\frac{1}{3} a \sigma^{3}-\frac{1}{4} b \sigma^{4}+\frac{1}{2} m_{\omega}^{2} \omega^{2}+\frac{1}{2} m_{\rho}^{2} \rho^{2},
\end{gathered}
$$




$$
\begin{gathered}
\varepsilon=\sum_{i=n, p} 2 \int \frac{\mathrm{d}^{3} k}{(2 \pi)^{3}} E_{i}^{\star}(k)\left[n^{q}\left(k, \mu_{i}^{\star}\right)+n^{q}\left(k,-\mu_{i}^{\star}\right)\right]+\frac{1}{2} m_{\sigma}^{2} \sigma^{2} \\
+\frac{1}{3} a \sigma^{3}+\frac{1}{4} b \sigma^{4}+\frac{1}{2} m_{\omega}^{2} \omega^{2}+\frac{1}{2} m_{\rho}^{2} \rho^{2},
\end{gathered}
$$

where $n\left(k, \mu_{i}\right)$ and $n\left(k,-\mu_{i}\right)$ are the fermion particle and antiparticle $q$-deformed distribution [8]

$$
n(k, \mu)=\frac{1}{[1+(q-1)(E(k)-\mu) / T]^{1 /(q-1)}+1} .
$$

The nucleon effective energy is defined as $E_{i}^{\star}=\sqrt{k^{2}+M_{i}^{\star 2}}$, where $M_{i}^{\star}=M_{N}-g_{\sigma} \sigma$. The effective chemical potentials $\mu_{i}^{\star}$ are given in terms of the vector meson mean fields $\mu_{i}=\mu_{i}^{\star}-g_{\omega} \omega \mp$ $g_{\rho} \rho$ (- proton, + neutron), where $\mu_{i}$ are the thermodynamical chemical potentials $\mu_{i}=\partial \varepsilon / \partial \rho_{i}$. At zero temperature they reduce to the Fermi energies $E_{F i} \equiv \sqrt{k_{F i}^{2}+M_{i}^{\star 2}}$ and the nonextensive statistical effects disappear. The isoscalar and isovector meson fields $(\sigma, \omega$ and $\rho)$ are obtained as a solution of the field equations in mean field approximation and the related couplings $\left(g_{\sigma}, g_{\omega}\right.$ and $g_{\rho}$ ) are the free parameter of the model [11]. Finally, The baryon densities $\rho_{B}$ are given by

$$
\rho_{B}=\gamma \int \frac{\mathrm{d}^{3} k}{(2 \pi)^{3}}\left[n\left(k, \mu_{i}^{\star}\right)-n\left(k,-\mu_{i}^{\star}\right)\right]
$$

where $\gamma$ is the spin/isospin multiplicity.

Note that statistical mechanics enter as an external ingredient in the functional form of the "free" particle distribution of Eq.(2.3). Because of all the equations must be solved in a selfconsistent way, the presence of nonextensive statistical effects in the particle distribution function influence the many-body interaction in the self-obtained meson fields.

In Fig. 1, we report the obtained hadronic EOS: pressure as a function of the baryon number density for different values of $q$. Because of previous phenomenological studies in heavy-ion collisions have brought to values of $q$ greater than unity [2], we concentrate our analysis to $q>1$. The results are plotted at fixed value of $Z / A=0.4$. The range of the considered baryon density and the chosen values of the parameters correspond to a physical situation that can realized in the recently proposed high energy heavy-ion collisions experiment (see, for example, Ref.[13]).

\subsection{Nonextensive QGP equation of state}

In the simple model of free quarks in a bag [14], the pressure, energy density and baryon number density for a Fermi gas of quarks in the framework of nonextensive statistics can be written, respectively, as

$$
\begin{aligned}
& P=\sum_{f=u, d} \frac{1}{3} \frac{\gamma_{f}}{2 \pi^{2}} \int_{0}^{\infty} k \frac{\partial \varepsilon_{f}}{\partial k}\left[n^{q}\left(k, \mu_{f}\right)+n^{q}\left(k,-\mu_{f}\right)\right] k^{2} d k-B, \\
& \varepsilon=\sum_{f=u, d} \frac{\gamma_{f}}{2 \pi^{2}} \int_{0}^{\infty} \varepsilon_{f}\left[n^{q}\left(k, \mu_{f}\right)+n^{q}\left(k,-\mu_{f}\right)\right] k^{2} d k+B, \\
& \rho=\sum_{f=u, d} \frac{1}{3} \frac{\gamma_{f}}{2 \pi^{2}} \int_{0}^{\infty}\left[n\left(k, \mu_{f}\right)-n\left(k,-\mu_{f}\right)\right] k^{2} d k
\end{aligned}
$$




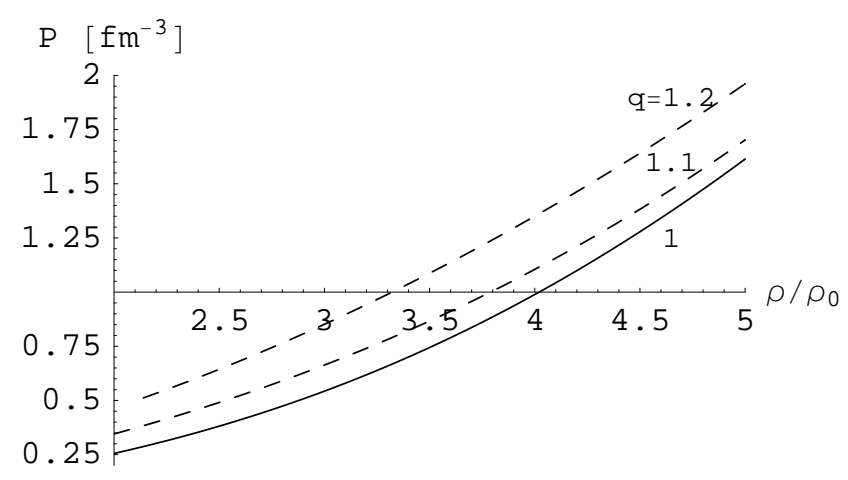

Figure 1: Hadronic equation of state: pressure versus baryon number density (in units of the nuclear saturation density $\rho_{0}$ ) for different values of $q$.

where $\varepsilon_{f}=\left(k^{2}+m_{f}^{2}\right)^{1 / 2}, n\left(k, \mu_{f}\right)$ and $n\left(k, \mu_{f}\right)$ are the particle and antiparticle quark distributions. The quark degeneracy for each flavor is $\gamma_{f}=6$. Similar expressions for the pressure and the energy density can be written for the gluons as massless Bose gas with zero chemical potential and degeneracy factor $\gamma_{g}=16$. Because of the fermion-boson nonextensive distribution (2.3), the results are not analytical, also in the massless quark approximation. Therefore, a numerical evaluations of the integral must be performed. Note that a similar calculation, only for the quark-gluon phase, was also performed in Ref.[15] by studying the phase transition diagram.

In Fig. 2, we report the EOS for massless quark $u, d$ and gluons, for different values of $q$. As in Fig. 1, the results are plotted at fixed value of $Z / A=0.4$; the bag parameter is $B^{1 / 4}=170 \mathrm{MeV}$. In both the figures we can observe remarkable effects in the shape of the EOS for small deviations from the standard statistics.

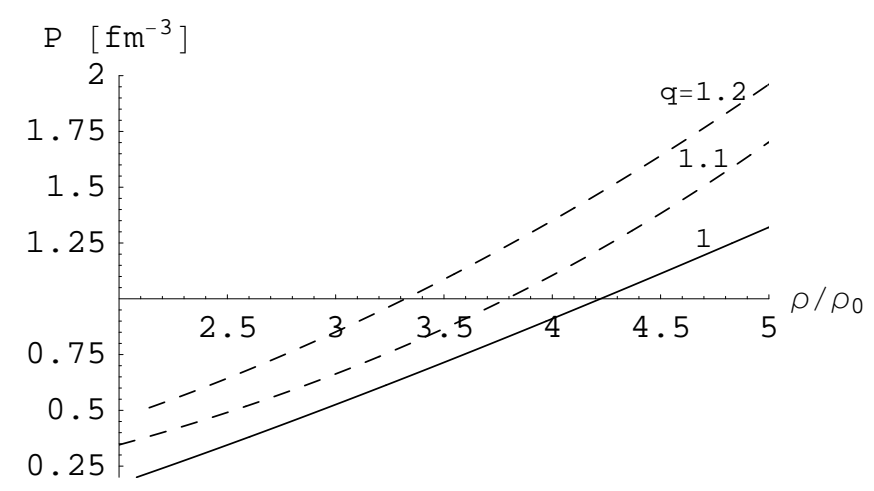

Figure 2: Same of Fig. 1 for the case of the quark-gluon equation of state. 


\section{Conclusion}

In this work we have studied the relativistic thermodynamic relations and derived the EOS of a gas of free particle at the equilibrium in the framework of the nonextensive Tsallis thermostatistics. The results are applied to obtain a consistent generalization of the EOS of strongly interacting hadronic matter and of deconfined QGP. The range of density and temperature chosen are physical values estimated in the recently proposed high energy heavy-ion collisions experiments at finite baryon chemical potential [13]. We find that small deviations from the Boltzmann-Gibbs statistics implies a sensible modification of the two considered EOSs. A complete discussion on the nuclear astrophysics consequence lies out the scope of this paper, however we want to outline that such a modification of the EOSs can strongly affect different physical properties, like, for example, the critical phase transition density, the symmetry energy, the nuclear compressibility, connected to experimental observables.

\section{References}

[1] C. Tsallis, J. Stat. Phys. 52, 479 (1988). See also: Nonextensive thermodynamic and physical applications, special number of Physica A 305 (2002) N. 1-2.

[2] W.M. Alberico, A. Lavagno, P. Quarati, Eur. Phys. J. C 12 (2000) 499; A. Lavagno, P. Quarati, Phys. Lett. B 498 (2001) 47.

[3] G. Wilk, Z. Wlodarczyk, Phys. Rev. Lett. 84 (2000) 2770.

[4] D.B. Walton, J. Rafelski, Phys. Rev. Lett. 84 (2000) 31.

[5] I. Bediaga, E.M.F. Curado, J.M. de Miranda, Physica A 286 (2000) 156.

[6] C. Beck, Physica A 305 (2002) 209.

[7] D.B. Ion, M.L.D. Ion, Phys. Lett. B 519 (2001) 63.

[8] A. Lavagno, Physics Letters A 301 (2002) 13.

[9] S.R. Groot, W.A. van Leeuwen, Ch. G. van Weert, Relativistic kinetic theory, North-Holland, Amsterdam, 1980.

[10] U. Tirnakli, F. Büyükkiliç, D. Demirhan, Phys. Lett. A 245 (1998) 62.

[11] N.K. Glendenning, S.A. Moszkowski, Phys. Rev. Lett. 67 (1991) 2414. J.D. Walecka, Ann. of Phys. 83 (1974) 491.

[12] J. Boguta, A.R. Bodmer, Nucl. Phys. A 292 (1977) 413.

[13] GSI, Scientific Report 2002 and GSI Proposal for an International Accelerator Facility for Research with Ions and Antiprotons, November 2001.

[14] A. Chodos et al., Phys. Rev. D 9 (1974) 3471.

[15] A. Teweldeberhan, H.G. Miller, R. Tegen, Int. J. Mod. Phys. E 12 (2003) 395. 